\title{
Disseminated Histoplasmosis: A Rare Case Report
}

\author{
Meenu Roat ${ }^{1}$, Jyotirmaya Gohli ${ }^{2}$, Suresh Chandra Gupta ${ }^{3}$
}

\begin{abstract}
Progressive disseminated histoplasmosis is a rare fungal infection and occurs in immunocompromised and immunosuppressed patients in the endemic area. It is difficult to differentiate it from tuberculosis on the basis of imaging finding alone. Here, we report a biopsy proven case of bilateral adrenal histoplasmosis with involvement of central nervous system (CNS). This patient showed good response to antifungal treatment. Keywords: Adrenal, Computed tomography, Disseminated histoplasmosis, Immunocompromised, Magnetic resonance imaging. Journal of Mahatma Gandhi University of Medical Sciences \& Technology (2018): 10.5005/jp-journals-10057-0085
\end{abstract}

\section{INTRODUCTION}

Histoplasmosis occurs due to a dimorphic fungus, histoplasma capsulatum which grows in soil fertilized by excreta of birds and bats. Humans are infected by inhalation of spores. They are converted into pathogenic yeast form in the alveolar macrophages from where they disseminate hematogenous to multiple organs, brain and spinal cord. Progressive histoplasmosis usually occurs in immunosuppressed individual due to failure of clearance of infection. CNS involvement is seen in only $10-20 \%$ of cases of progressive histoplasmosis. Clinical presentation of disseminated histoplasmosis is nonspecific and resembles chronic infection or malignancies.

\section{Case Description}

A 50-year-old male patient presented to our hospital with complaints of pain in the abdomen, low-grade intermittent fever, drowsiness, uneasiness and anorexia for the last 3 months. The patient was well 3 months back when he developed the above symptoms with gradual progression. The patient was a known case of type ll diabetes. Contrast enhanced computed tomography (CECT) abdomen was performed which was suggestive of hepatosplenomegaly and enlarged bilateral adrenal gland, with peripheral rim enhancement and enhancing internal septations (Figs 1 to 3 ).

Differentials of enlarged bilateral adrenal gland with peripheral rim and enhancing septations include granulomatous lesion, metastasis and bilaterally pheochromocytoma.

Magnetic resonance imaging of the brain revealed multiple ring enhancing lesions in the left middle cerebral peduncle, right cerebellar hemisphere and right frontal lobe. The center of the lesion shows hypointense center on T2W images (Figs 4 and 5). Mild to moderate perilesional edema was seen suggestive of granulomatous lesion. Another possibility of metastasis cannot be ruled out entirely (Figs 6 and 7).

\section{Discussion}

Histoplasmosis is a self-limiting pulmonary infection that is usually asymptomatic or may presents as a flu-like illness. There are three major clinical presentations of histoplasmosis, i.e., pulmonary form, progressive disseminated form, and primary cutaneous histoplasmosis. H. capsulatum disseminates homogenously during a primary episode of infection to extra-pulmonary sites. However,
${ }^{1-3}$ Department of Radiodiagnosis, Mahatma Gandhi Medical College and Hospital, Mahatma Gandhi University of Medical Sciences and Technology, Jaipur, Rajasthan, India

Corresponding Author: Meenu Roat, Department of Radiodiagnosis, Mahatma Gandhi Medical College and Hospital, Mahatma Gandhi University of Medical Sciences and Technology, Jaipur, Rajasthan, India, Phone: +91 8619966400, e-mail: meenuroat123@gmail.com

How to cite this article: Roat M, Gohli J, Gupta SC. Disseminated Histoplasmosis: A Rare Case Report. J Mahatma Gandhi Univ Med Sci Tech 2018;3(3):99-101.

Source of support: Nil

Conflict of interest: None

clinical features of progressive disseminated histoplasmosis usually occur in extreme of ages or in immunocompromised state.

The common abdominal findings of disseminated histoplasmosis (DH) as described by many authors on imaging includes mild to moderate hepatosplenomegaly without focal hypodense lesion in the spleen. ${ }^{1-5}$ The patient usually reveals a bilateral adrenal mass of varied features. On CT, symmetrical enlargement of the adrenal gland with preservation of its contours, central hypodensity with peripheral enhancement and presence of calcification is seen. ${ }^{1,4}$

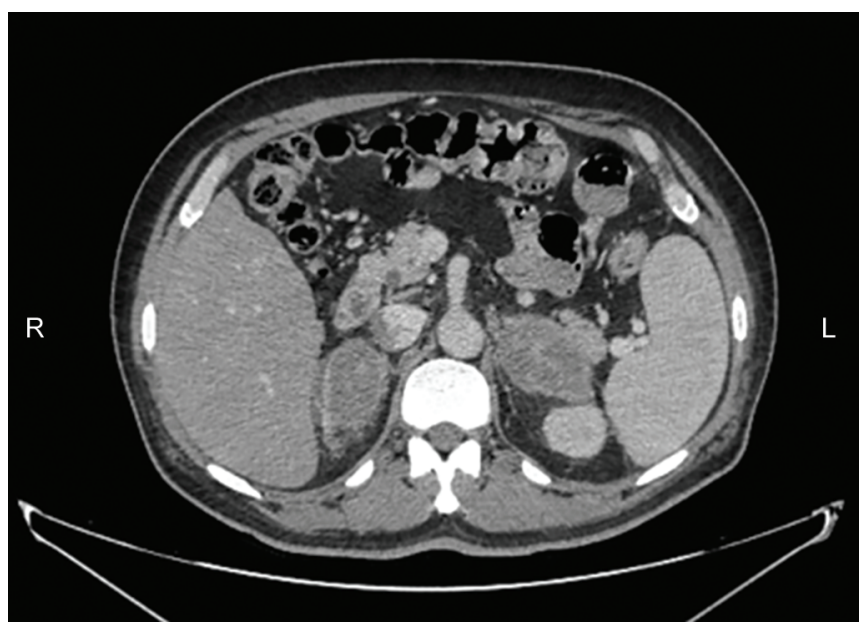

Fig. 1: Contrast enhanced CT of the abdomen at the level of adrenal gland shows enlarged bilateral adrenal gland with peripheral rim enhancement and enhancing internal septations 


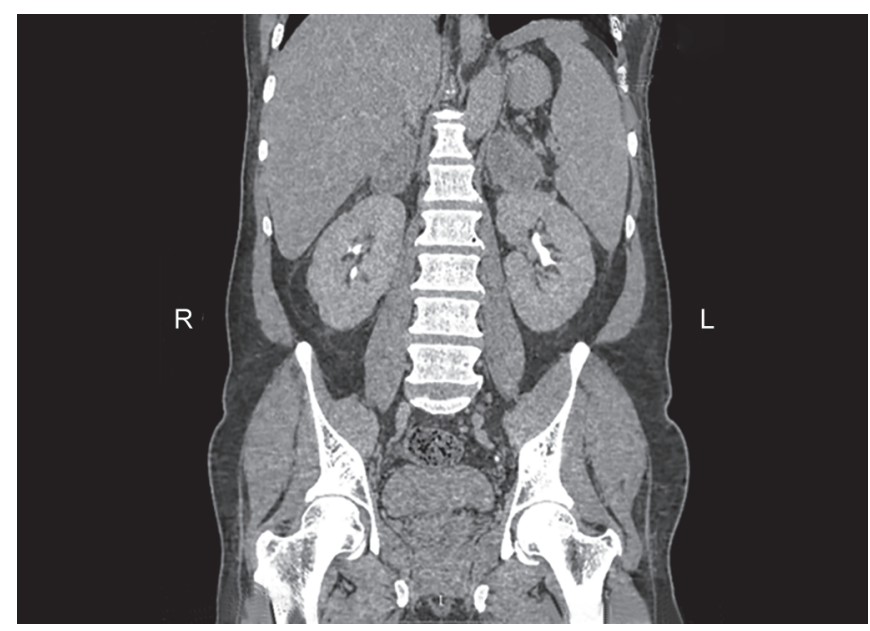

Fig. 2: Sagittal CT of the abdomen showing hepatosplenomegaly, enlarged bilateral adrenal gland with central hypoattenuating area suggestive of necrosis

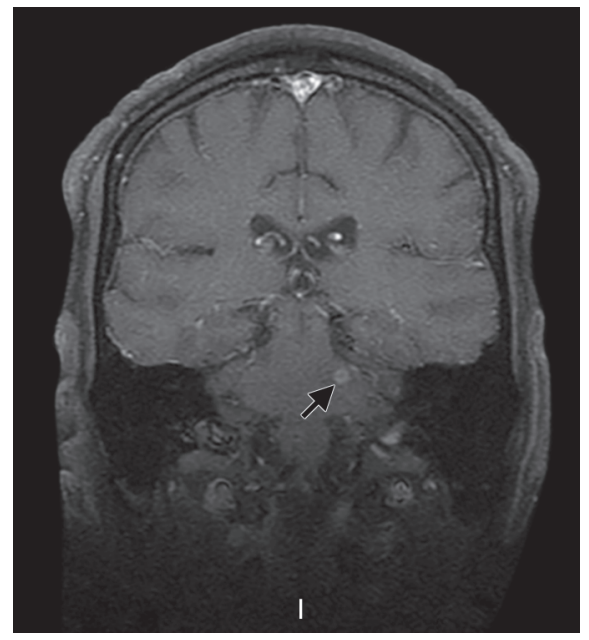

Fig. 4: Contrast-enhancedT1W images of the patient showing multifocal rounded ring enhancing lesions in left cerebellar peduncle

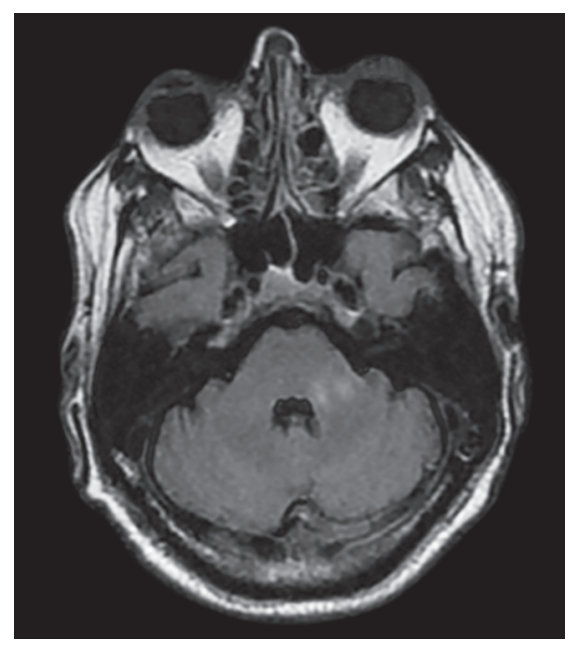

Fig. 6: T2 FLAIR MRI revealing mild perilesional edema in left middle cerebellar peduncle

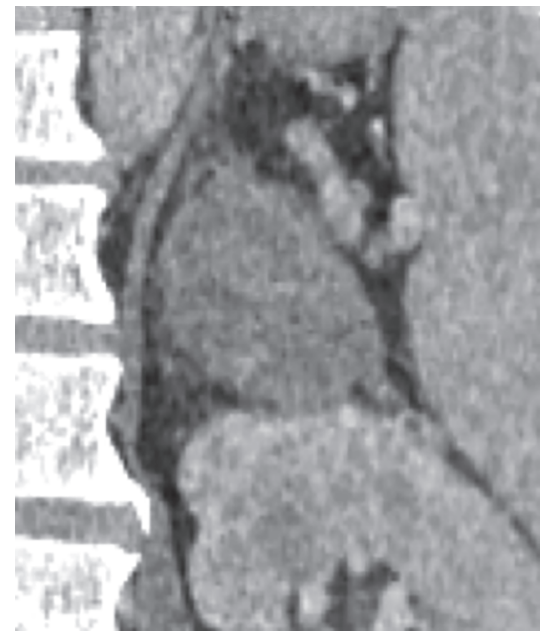

Fig. 3: Sagittal CT of the abdomen showing enlarged bilateral adrenal gland with central hypoattenuating area suggestive of necrosis

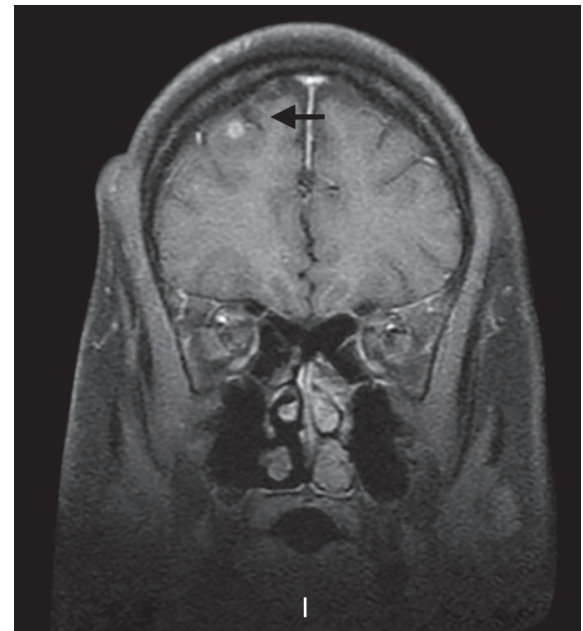

Fig. 5: Contrast-enhancedT1W images of the patient showing multifocal rounded ring enhancing lesions in right frontal lobe

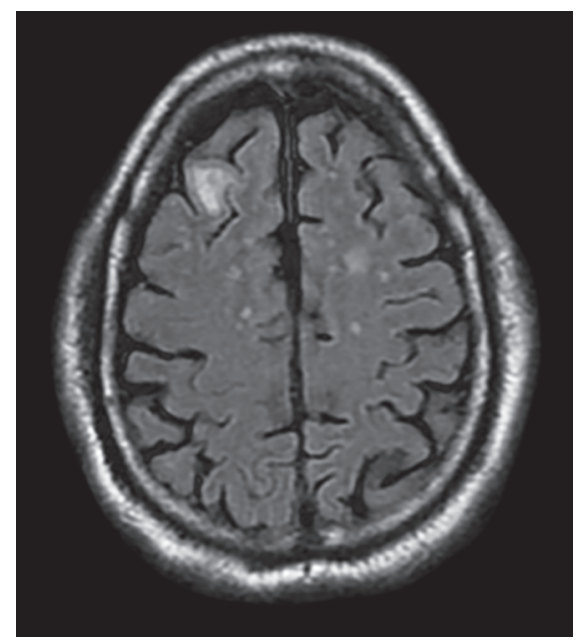

Fig. 7: T2 FLAIR MRI revealing mild perilesional edema in bilateral cerebral hemisphere 
In our patient we observed hepatosplenomegaly without any focal lesion in spleen and enlarged bilateral adrenal glands with peripheral enhancing rim and enhancing internal septations.

The involvement of CNS is seen in only $5-20 \%$ of patients of the cases of $\mathrm{DH}$ and is more commonly seen in immunosuppresed individuals. ${ }^{6} \mathrm{DH}$, especially with ring enhancing lesions are rarely seen in immunocompetent individuals. In our patient, multifocal rounded ring enhancing lesion with mild to moderate perilesional edema is seen on MRI.

Differentials based on imaging include bilateral adrenal granulomatous lesion with granules in the brain or bilateral the adrenal lesion with metastasis in brain. Computed tomography (CT) guided biopsy of adrenal lesion was done and it came out positive for histoplasmosis. The patient was treated with antifungal drugs and the patient showed a good clinical response. On follow-up after 1-year, a significant reduction of the size of lesions was seen.

In conclusion, it is difficult to differentiate histoplasmosis from tuberculosis on imaging alone and biopsy is needed for confirmation of diagnosis.

\section{References}

1. Grover S, Midha N, Gupta M, et al. Imaging spectrum in disseminated histoplasmosis: case report and brief review. Australas Radiol 2005;49(2):175-178. DOI: 10.1111/j.1440-1673.2005.01369.x.

2. Kawashima A, Sandler CM, Fishman EK, et al. Spectrum of CT findings in nonmalignant disease of the adrenal gland. Radiographics 1998;18(2):393-412. DOI: 10.1148/radiographics.18.2.9536486.

3. Gibb WRG, Ramsay AD, McNeil NI, et al. Bilateral adrenal masses. Br Med J (Clin Res Ed) 1985;291(6489):203-204. DOI: 10.1136/ bmj.291.6489.203.

4. Radin DR. Disseminated histoplasmosis: abdominal CT findings in 16 patients. AJR Am J Roentgenol 1991;157(5):955-958. DOI: 10.2214/ ajr.157.5.1927816.

5. Starkey J, Moritani T, Kirby P. MRI of CNS fungal infections: review of aspergillosis to histoplasmosis and everything in between. Clin Neuroradiol 2014;24(3):217-230. DOI: 10.1007/s00062-0140305-7.

6. Saccente M, McDonnell RW, Baddour LM, et al. Cerebral histoplasmosis in the azole era: report of four cases and review. South Med J 2003;96(4):410-416. DOI: 10.1097/01.SMJ.0000051734.53654.D0. 